$11-1-2010$

\title{
A Comparison between Unbiased Ridge and Least Squares Regression Methods Using Simulation Technique
}

Mowafaq M. Al-Kassab

Al-al Bayt University, Mafraq, Jordan, omar_qwaider_81@yahoo.com

Omar Q. Qwaider

Al-al Bayt University, Mafraq, Jordan, mowafaq2002@yahoo.co.uk

Follow this and additional works at: http://digitalcommons.wayne.edu/jmasm

Part of the Applied Statistics Commons, Social and Behavioral Sciences Commons, and the Statistical Theory Commons

\section{Recommended Citation}

Al-Kassab, Mowafaq M. and Qwaider, Omar Q. (2010) "A Comparison between Unbiased Ridge and Least Squares Regression Methods Using Simulation Technique," Journal of Modern Applied Statistical Methods: Vol. 9 : Iss. 2 , Article 16.

DOI: $10.22237 /$ jmasm/1288584900

Available at: http://digitalcommons.wayne.edu/jmasm/vol9/iss2/16 


\title{
A Comparison between Unbiased Ridge and Least Squares Regression Methods Using Simulation Technique
}

\author{
Mowafaq M. Al-Kassab Omar Q. Qwaider \\ Al-al Bayt University, \\ Mafraq, Jordan
}

The parameters of the multiple linear regression are estimated using least squares $\left(\hat{B}_{L S}\right)$ and unbiased ridge regression methods $(\hat{B}(K I, J))$. Data was created for fourteen independent variables with four different values of correlation between these variables using Monte Carlo techniques. The above methods were compared using the mean squares error criterion. Results show that the unbiased ridge method is preferable to the least squares method.

Key words: Least squares, prior information, unbiased ridge estimation, mean squares error.

Introduction

Consider the linear regression model:

$$
Y^{*}=X^{*} B+U
$$

where $X^{*}$ is a $(n \times(p+1))$ matrix of predictor variables of full rank, $Y^{*}$ is a $(n \times 1)$ response vector, $B$ is a $((p+1) \times 1)$ vector of parameters and $U$ is a $(n \times 1)$ vector of errors with $E(U)=0 \quad$ and $\quad \operatorname{Cov}(U)=\sigma^{2} I . \quad$ When multicollinearity exists, the least squares estimate $\hat{B}_{\mathrm{LS}}=\left(\mathrm{X}^{* \mathrm{~T}} \mathrm{X}^{*}\right)^{-1} \mathrm{X}^{* \mathrm{~T}} \mathrm{Y}^{*}$ is unstable, and many different methods have been proposed to control multicollinearity (Hoerl \& Kennard, 1970).

M. M. T. Al-Kassab is a Professor of Mathematical Statistics and Deputy Dean of the College of Science. His research interests include optimum stratum boundaries and biased and unbiased methods in regression. Email: mowafaq2002@yahoo.co.uk. Omar Q. Qwaider has his M.Sc. in statistics. His research interests are in regression estimation methods. Email: omar_qwaider_81@yahoo.com.
An alternative to the linear regression method is the unbiased ridge estimate

$$
\hat{B}(K I, J)=\left(X^{* T} X^{*}+K I_{P}\right)^{-1}\left(X^{* T} Y^{*}+K J\right)
$$

where

$$
J=\frac{\sum_{i=1}^{P} \hat{B}_{i l s}}{P}
$$

and

$$
\hat{K}=\frac{P \sigma^{2}}{(\hat{B}-J)^{T}(\hat{B}-J)-\sigma^{2} \operatorname{tr}\left(X^{T} X\right)^{-1}} .
$$

The unbiased ridge estimate regression, $\hat{B}(K I, J)$, has advantages and disadvantages. It is effective in practice but it is a complicated function of $K$, thus it is necessary to use rather complicated equations when employing some popular methods such as the Crouse, Jin and Hanumare (1995) criterion to select $K$ (Swindel, 1976).

The General Multiple Linear Regression Model The general multiple linear regression model is 


\section{AL-KASSAB \& QWAIDER}

$$
\begin{gathered}
\mathrm{Y}_{\mathrm{i}}^{*}=\mathrm{B}_{0}+\mathrm{B}_{1} \mathrm{X}_{\mathrm{i} 1}^{*}+\mathrm{B}_{2} \mathrm{X}_{\mathrm{i} 2}^{*}+\ldots+\mathrm{B}_{\mathrm{p}} \mathrm{X}_{\mathrm{ip}}^{*}+\mathrm{U}_{\mathrm{i}} \\
\mathrm{i}=1,2, \ldots, \mathrm{n}
\end{gathered}
$$

where $B_{0}, B_{1}, B_{2}, \ldots ., B_{P}$ are the regression coefficients and $U_{i} \sim N\left(0, \sigma^{2}\right)$ is the random error associated with the observations. In matrix notation model (2-1) can be written as

$$
\begin{gathered}
{\left[\begin{array}{l}
y_{1}^{*} \\
y_{2}^{*} \\
\vdots \\
y_{n}^{*}
\end{array}\right]=\left[\begin{array}{ccccc}
1 & x_{11}^{*} & x_{12}^{*} & \ldots & x_{1 p}^{*} \\
1 & x_{21}^{*} & x_{22}^{*} & \ldots & x_{2 p}^{*} \\
\vdots & \vdots & \vdots & \ddots & \vdots \\
1 & x_{n 1}^{*} & x_{n 2}^{*} & \ldots & x_{n p}^{*}
\end{array}\right]\left[\begin{array}{l}
B_{0} \\
B_{1} \\
\vdots \\
B_{p}
\end{array}\right]+\left[\begin{array}{l}
U_{1} \\
U_{2} \\
\vdots \\
U_{n}
\end{array}\right]} \\
Y^{*}=X^{*} B+U,
\end{gathered}
$$

where $Y^{*}$ is a $(n \times 1)$ column vector of observations on the dependent variable, $X^{*}$ is a $((p+1) \times 1)$ matrix resulting from $n$ observations on $P$ explanatory variables $X_{1}^{*}, X_{2}^{*}, \ldots . ., X_{p}^{*}$ where the first column of 1's represent the intercept term, that is, $X_{0}^{*}=1$, and $U \sim N\left(0, \sigma^{2}\right)$ is $(n \times 1)$ column vector of errors.

Assumptions of the standardized model are:

$$
\begin{aligned}
& \text { 1. } E(U)=0 \\
& \text { 2. } \operatorname{Var}(U)=E\left(U U^{T}\right)=\sigma^{2} I \\
& \text { 3. } \operatorname{Rank}\left(X^{*}\right)=\mathrm{P} \text { where } p<n
\end{aligned}
$$

The ordinary least squares estimators are given by $\hat{B}_{\mathrm{LS}}=\left(\mathrm{X}^{* \mathrm{~T}} \mathrm{X}^{*}\right)^{-1} \mathrm{X}^{* \mathrm{~T}} \mathrm{Y}^{*}$.

Properties of Ordinary Least Squares Estimators

1. Unbiasedness:

An estimator, $\widehat{B}$, is said to be unbiased estimator of $B$ if the expected value of $\widehat{B}$ equals $B$, that is, $E\left(\hat{B}_{L S}\right)=B$. (Casella \& Berger, 2002)

2. Variance:

$$
\operatorname{Var}\left(\hat{B}_{L S}\right)=\sigma^{2}\left(X^{* T} X^{*}\right)^{-1}
$$

3. Mean squared error:

$$
\begin{aligned}
& \operatorname{MSE}(\hat{B})=\sum_{i=1}^{P} \operatorname{Var}\left(\hat{B}_{i}\right)+\sum_{i=1}^{P}\left(\operatorname{Bias}\left(\hat{B}_{i}\right)\right)^{2} \\
& \Rightarrow \operatorname{MSE}\left(\hat{B}_{L S}\right)=\sum_{i=1}^{P} \operatorname{Var}\left(\hat{B}_{i}\right)=\sigma^{2} \operatorname{tr}\left(X^{T} X\right)^{-1} \\
& \operatorname{MSE}\left(\hat{B}_{L S}\right)=\sigma^{2} \sum_{i=1}^{P} \frac{1}{\ell_{i}}
\end{aligned}
$$

\section{Unbiased Ridge Estimator}

Ridge regression, which was proposed by Horel and Kennard (1970), suggests the use of $X^{T} X+K$, where $K$ is a diagonal matrix rather than $X^{T} X$, so that the resulting estimators of $B$ are known as the ridge regression estimators and are given by:

$$
\hat{B}=\left(X^{T} X+K\right)^{-1} X^{T} Y
$$

Horel and Kennard (1970) suggested two forms for $K$. First, if $\mathrm{K}=\mathrm{kI}_{\mathrm{p}}, 0<\mathrm{k}<1$. Substituting this in equation (3.1), results in

$$
\hat{B}(k)=\left(X^{T} X+k I_{p}\right)^{-1} X^{T} Y
$$

and, using eigenvalues and eigenvectors, $\hat{B}(k)$ can be expressed as

$$
\hat{B}(k)=\sum_{j=1}^{p}\left(\ell_{j}+k\right)^{-1} V_{j} V_{j}^{T} X^{T} Y
$$

Second, if $\mathrm{K}=\operatorname{diag}\left(\mathrm{k}_{\mathrm{i}}\right), \mathrm{k}_{\mathrm{i}}>0 \mathrm{i}=1,2, \ldots ., \mathrm{p}$, then 


$$
\hat{B}(k)=\sum_{j=1}^{p}\left(\ell_{j}+k_{j}\right)^{-1} V_{j} V_{j}^{T} X^{T} Y
$$

Swindle (1976) illustrated a technique for combining prior information with ridge regression that extended Hoerl and Kennard's model as follows:

$$
B(k I, J)=\left(X^{T} X+k I\right)^{-1}\left(X^{T} Y+k J\right)
$$

with $J$ being a fixed vector of prior estimate of $B$. Swindle showed that there exists a value $\mathrm{k}$ which gives a smaller MSE than the least squares estimator for any fixed prior information, $J$.

Definition (1): A prior mean $J$ is said to be good if the difference $\operatorname{MSE}(\hat{\mathrm{B}}(\mathrm{K}))-\operatorname{MSE}(\hat{\mathrm{B}}(\mathrm{kI}, \mathrm{J}))$ is positive for all positive values $k$ when both $\hat{B}(k)$ and $\hat{B}(k I, J)$ are computed by using the same value of $k$ (Pliskin, 1987).

Remark: The restriction $k>0$ is made because, if $k=0$ then

$$
\hat{B}_{L S}=\hat{B}(k)=\hat{B}(k I, J)=\left(X^{T} X\right)^{-1} X^{T} Y
$$

for all $J$, thereby implying that all three estimators have the same risk. In this study, it was found that the vector of prior information $J$ depends on the arithmetic mean of the least squares estimators multiplying by a vector whose elements are ones, that is

$$
J=\left[\frac{\sum_{i=1}^{p} \hat{B}_{i L S}}{p}\right] I_{p \times 1}
$$

Unbiasedness of Ridge Estimators: Theorem (1)
Consider the standard linear regression model (2.1), where $U$ is normally distributed $N\left(0, \sigma^{2} I\right)$, and the least square estimator, $\hat{B}$ is normally distributed $N\left(B, \sigma^{2}\left(X^{T} X\right)^{-1}\right)$. The prior information $J$ is independent of $\hat{B}_{L S}$, and $J$ is normally distributed $N(B, V)$. Also assume that $V$ has full rank covariance matrix and that the convex estimator is $B(C, J)=C \hat{B}_{L S}+(I-C) J$, where $I$ is the $P \times P$ identity matrix and $C$ is a $P \times P$ matrix. The optimal $C$ in terms of minimum MSE is then

$$
\mathrm{C}=\mathrm{V}\left(\sigma^{2}\left(\mathrm{X}^{\mathrm{T}} \mathrm{X}\right)^{-1}+\mathrm{V}\right)^{-1}
$$

Corollary (1): Suppose $\hat{B}$ is an estimator of $B$ with mean $B$ and covariance matrix $\sum$, and $J$ is prior information with mean $B$ and covariance matrix $V$. Further assume that if $J$ is uncorrelated with $\hat{B}$, and $V$ and $\sum$ are of full rank, then the convex estimator $B(C, J)$ has a minimum MSE of optimal value

$$
C=V\left(V+\sum\right)^{-1}
$$

Theorem (2): Unbiased Ridge Estimate of $B$ (Crouse, et al., 1995)

Let $\hat{B}_{L S}$ have a distribution with mean $B$ and covariance $\sigma^{2}\left(\mathrm{X}^{\mathrm{T}} \mathrm{X}\right)^{-1}$, denoted by $N\left(B, \sigma^{2}\left(X^{T} X\right)^{-1}\right)$, as in the linear model. Similarly, let $J$ be distributed $\mathrm{N}\left(\mathrm{B},\left(\frac{\sigma^{2}}{\mathrm{k}}\right) \mathrm{I}\right)$ for $\mathrm{k}>0$, and define $\mathrm{B}(\mathrm{C}, \mathrm{J})=\mathrm{CB}_{\mathrm{LS}}+(\mathrm{I}-\mathrm{C}) \mathrm{J}$; then, for the optimal value $C$ in terms of minimum

MSE

$$
\mathrm{B}(\mathrm{C}, \mathrm{J})=\hat{\mathrm{B}}(\mathrm{kI}, \mathrm{J})=\left(\mathrm{X}^{\mathrm{T}} \mathrm{X}+\mathrm{kI}\right)^{-1}\left(\mathrm{X}^{\mathrm{T}} \mathrm{Y}+\mathrm{KJ}\right) \text {, }
$$
and $B(C, J)$ is an unbiased estimate of $B$. 
Proof: Assuming that $\mathrm{J} \sim \mathrm{N}\left(\mathrm{B},\left(\frac{\sigma^{2}}{\mathrm{k}}\right) \mathrm{I}\right)$ and, from corollary (1), $\hat{B}$ has a distribution with mean $B$ and covariance $\sum=\sigma^{2}\left(X^{\mathrm{T}} X\right)^{-1}$, that is, $\hat{B} \sim \mathrm{N}\left(\mathrm{B}, \sum\right)$, it is found that $J$ is distributed with mean $B$ and covariance $\mathrm{V}=\left(\frac{\sigma^{2}}{\mathrm{k}}\right) \mathrm{I}$ denoted by $\mathrm{J} \sim \mathrm{N}(\mathrm{B}, \mathrm{V})$. Substituting this into equation (3.8) results in

$$
\begin{aligned}
\hat{\mathrm{C}} & =\frac{\sigma^{2}}{\mathrm{k}}\left(\sigma^{2}\left(\mathrm{X}^{\mathrm{T}} \mathrm{X}\right)^{-1}+\frac{\sigma^{2}}{\mathrm{k}} \mathrm{I}\right)^{-1} \\
& =\frac{\mathrm{I}}{\mathrm{k}}\left(\left(\mathrm{X}^{\mathrm{T}} \mathrm{X}\right)^{-1}+\left(\frac{\mathrm{I}}{\mathrm{k}}\right) \mathrm{I}\right)^{-1} \\
& =\left[\mathrm{k}\left(\mathrm{X}^{\mathrm{T}} \mathrm{X}\right)^{-1}+\mathrm{I}\right]^{-1}
\end{aligned}
$$

Substituting $B(C, J)=\hat{C B}+(I-C) J$, results in

$$
\begin{aligned}
\mathrm{B}(\mathrm{C}, \mathrm{J})= & \left(\mathrm{k}\left(\mathrm{X}^{\mathrm{T}} \mathrm{X}\right)^{-1}+\mathrm{I}\right)^{-1}\left(\mathrm{X}^{\mathrm{T}} \mathrm{X}\right)^{-1} \mathrm{X}^{\mathrm{T}} \mathrm{Y} \\
& +\left(\mathrm{I}-\left(\mathrm{k}\left(\mathrm{X}^{\mathrm{T}} \mathrm{X}\right)^{-1}+\mathrm{I}\right)^{-1}\right) \mathrm{J}
\end{aligned}
$$

and

$$
\begin{aligned}
\mathrm{B}(\mathrm{C}, \mathrm{J})= & \left(\left(\mathrm{X}^{\mathrm{T}} \mathrm{X}\right)+\mathrm{kI}\right)^{-1} \mathrm{X}^{\mathrm{T}} \mathrm{Y} \\
& +\left(\mathrm{I}-\left(\mathrm{k}\left(\mathrm{X}^{\mathrm{T}} \mathrm{X}\right)^{-1}+\mathrm{I}\right)^{-1}\right) \mathrm{J}
\end{aligned}
$$

Multiplying $\left(\mathrm{K}\left(\mathrm{X}^{\mathrm{T}} \mathrm{X}\right)^{-1}+\mathrm{I}\right)^{-1}$ by $\mathrm{X}^{\mathrm{T}} \mathrm{X}\left(\mathrm{X}^{\mathrm{T}} \mathrm{X}\right)^{-1}$, results in

$$
\begin{aligned}
\mathrm{B}(\mathrm{C}, \mathrm{J})= & \left(\left(\mathrm{X}^{\mathrm{T}} \mathrm{X}\right)+\mathrm{kI}\right)^{-1} \mathrm{X}^{\mathrm{T}} \mathrm{Y}+ \\
& \left(\mathrm{I}-\mathrm{X}^{\mathrm{T}} \mathrm{X}\left(\mathrm{X}^{\mathrm{T}} \mathrm{X}\right)^{-1}\left(\mathrm{k}\left(\mathrm{X}^{\mathrm{T}} \mathrm{X}\right)^{-1}+\mathrm{I}\right)^{-1}\right) \mathrm{J} \\
= & \left(\left(\mathrm{X}^{\mathrm{T}} \mathrm{X}\right)+\mathrm{kI}\right)^{-1} \mathrm{X}^{\mathrm{T}} \mathrm{Y} \\
& +\left(\mathrm{I}-\mathrm{X}^{\mathrm{T}} \mathrm{X}\left(\left(\mathrm{X}^{\mathrm{T}} \mathrm{X}\right)+\mathrm{kI}\right)^{-1}\right) \mathrm{J}
\end{aligned}
$$

Adding and subtracting $k I$ to $X^{T} X$,

$$
\begin{aligned}
\mathrm{B}(\mathrm{C}, \mathrm{J}) & =\left(\begin{array}{l}
\left(\mathrm{X}^{\mathrm{T}} \mathrm{X}+\mathrm{kI}\right)^{-1} \mathrm{X}^{\mathrm{T}} \mathrm{Y}+ \\
\left(\mathrm{I}-\left(\mathrm{X}^{\mathrm{T}} \mathrm{X}+\mathrm{kI}-\mathrm{kI}\right)\left(\mathrm{X}^{\mathrm{T}} \mathrm{X}+\mathrm{kI}\right)^{-1}\right) \mathrm{J}
\end{array}\right) \\
& =\left(\begin{array}{l}
\left(\mathrm{X}^{\mathrm{T}} \mathrm{X}+\mathrm{kI}\right)^{-1} \mathrm{X}^{\mathrm{T}} \mathrm{Y}+ \\
\left(\mathrm{I}+\mathrm{k}\left(\mathrm{X}^{\mathrm{T}} \mathrm{X}+\mathrm{kI}\right)^{-1}-\mathrm{I}\right) \mathrm{J}
\end{array}\right)
\end{aligned}
$$

Simplifying the above results in:

$$
\begin{aligned}
\mathrm{B}(\mathrm{C}, \mathrm{J}) & =\hat{\mathrm{B}}(\mathrm{kI}, \mathrm{J}) \\
& =\left(\mathrm{X}^{\mathrm{T}} \mathrm{X}+\mathrm{kI}\right)^{-1}\left(\mathrm{X}^{\mathrm{T}} \mathrm{Y}+\mathrm{kJ}\right)
\end{aligned}
$$

Swindle (1976) did not propose a method for estimating the parameter $k$, however, Crouse, et al. (1995) proposed a procedure to estimate $\mathrm{k}$, as follows:

$$
\hat{k}=\left\{\begin{array}{l}
\frac{P \sigma^{2}}{(\hat{B}-J)^{T}(\hat{B}-J)-\sigma^{2} \operatorname{tr}\left(X^{T} X\right)^{-1}}, \\
\text { if }(\hat{B}-J)^{T}(\hat{B}-J)-\sigma^{2} \operatorname{tr}\left(X^{T} X\right)^{-1}>0 \\
\frac{P \sigma^{2}}{(\hat{B}-J)^{T}(\hat{B}-J)}, \quad \text { o.w. }
\end{array}\right.
$$

If $\sigma^{2}$ is unknown, then $\sigma^{2}$ can be estimated by an unbiased estimator, 


$$
\hat{\mathrm{k}}=\left\{\begin{array}{l}
\frac{\mathrm{Ps}^{2}}{(\hat{\mathrm{B}}-\mathrm{J})^{\mathrm{T}}(\hat{\mathrm{B}}-\mathrm{J})-\mathrm{s}^{2} \operatorname{tr}\left(\mathrm{X}^{\mathrm{T}} \mathrm{X}\right)^{-1}}, \\
\text { if }(\hat{\mathrm{B}}-\mathrm{J})^{\mathrm{T}}(\hat{\mathrm{B}}-\mathrm{J})-\sigma^{2} \operatorname{tr}\left(\mathrm{X}^{\mathrm{T}} \mathrm{X}\right)^{-1}>0 \\
\frac{\mathrm{Ps}^{2}}{(\hat{\mathrm{B}}-\mathrm{J})^{\mathrm{T}}(\hat{\mathrm{B}}-\mathrm{J})}, \text { o.w. }
\end{array}\right.
$$

Properties of the Unbiased Ridge Estimators

1. Unbiasedness:

$$
\mathrm{E}(\hat{\mathrm{B}}(\mathrm{kI}, \mathrm{J}))=\mathrm{B}
$$

2. Variance:

$$
\operatorname{Var}\left(\hat{B}_{\mathrm{i}}(\mathrm{kI}, \mathrm{J})\right)=\sigma^{2} \frac{\ell_{\mathrm{i}}}{\left(\ell_{\mathrm{i}}+\mathrm{k}_{\mathrm{i}}\right)^{2}}
$$

3. Mean Square's Error:

$$
\begin{aligned}
& \operatorname{MSE}(\hat{B}(k I, J))= \\
& \sum_{i=1}^{p} \frac{I}{\left(\ell_{i}+k_{i}\right)^{2}}\left(\sum_{i=1}^{p} k_{i}\left(B_{i}-J\right)\right)^{2}+\sigma^{2} \sum_{i=1}^{p} \frac{\ell_{i}}{\left(\ell_{i}+k_{i}\right)^{2}}
\end{aligned}
$$

\section{Methodology}

Model Description and Monte Carlo Simulation

This research used a Monte Carlo study to examine the properties of least squares and unbiased ridge methods. The properties were then compared in the sense of the MSE, which was evaluated using equations (2.2) and (3.12) respectively. Thirty observations $(n=30)$ were generated for each of fourteen $(p=14)$ explanatory variables; the explanatory variables were generated using the device:

$$
\left\{\begin{array}{l}
X_{i j}^{*}=\left(1-\alpha^{2}\right)^{1 / 2} Z_{i j}^{*}+\alpha Z_{i 15}^{*}(j=1,2, \ldots, m . i=1,2, \ldots, 30) \\
X_{i j}^{*}=Z_{i j}^{*}(j=m+1, m+2, \ldots, 14 . i=1,2, \ldots, 30)
\end{array}\right.
$$

Where $Z_{\mathrm{ij}}$ are independent standard normal pseudo-random numbers, $Z_{i 15}$ is the $i^{\text {th }}$ element of the column vector of random error $Z_{15}, \alpha$ is specified so that the correlation between any two explanatory variables is given by $\alpha^{2}$. The $n$ observations for the dependent variable $\mathrm{Y}$ are determined by:

$$
\begin{aligned}
& \mathrm{Y}_{\mathrm{i}}=\lambda_{1} \mathrm{X}_{\mathrm{i} 1}+\lambda_{2} \mathrm{X}_{\mathrm{i} 2}+\ldots . .+\lambda_{14} \mathrm{X}_{\mathrm{i} 14}+\mathrm{U}_{\mathrm{i}} \\
& \mathrm{i}=1,2, \ldots ., 30
\end{aligned}
$$

where $U_{i}$ are independent normal $\left(0, \sigma^{2}\right)$ pseudo-numbers evaluated by: $U_{i}=Z_{i 15}-\bar{Z}_{15}$, and $\mathrm{Y}$ is standardized using unit length scale.

\section{Results}

The primary purpose of this research was to compare the MSE of the considered estimators, thus, the MSE for all estimators was evaluated. In addition, the efficiency of each estimator was evaluated. Thirteen experiments using Monte Carlo methods were conducted. The results of each experiment consist of five tables. The tables display the MSE of each estimator under one of five levels of correlation between explanatory variables. One set of experimental results is presented and consists of tables displaying the MSE of the least square and unbiased ridge methods for the desired correlation coefficients.

\section{Conclusion}

As shown in Tables 1-5, based on the thirteen experiments, it is concluded that the unbiased ridge method is preferable to the least square method because it results in smaller MSE values. 


\section{AL-KASSAB \& QWAIDER}

Table 1: Correlation Coefficient $\alpha^{2}=0.35$

\begin{tabular}{|c|c|c|}
\hline $\begin{array}{c}\text { Correlation } \\
\text { Between }\end{array}$ & $\begin{array}{c}\text { MSE Using } \\
\text { Least Squares }\end{array}$ & $\begin{array}{c}\text { MSE Using } \\
\text { Unbiased Ridge }\end{array}$ \\
\hline $\mathrm{X} 1, \mathrm{X} 2$ & 25.4000 & 0.122822 \\
\hline $\mathrm{X} 1-\mathrm{X} 3$ & 23.1838 & 0.0039352 \\
\hline $\mathrm{X} 1-\mathrm{X} 4$ & 30.7029 & 0.0368124 \\
\hline $\mathrm{X} 1-\mathrm{X} 5$ & 36.5401 & 0.144270 \\
\hline $\mathrm{X} 1-\mathrm{X} 6$ & 28.5695 & 0.0714341 \\
\hline $\mathrm{X} 1-\mathrm{X} 7$ & 25.3975 & 0.0241636 \\
\hline $\mathrm{X} 1-\mathrm{X} 8$ & 36.4954 & 0.128423 \\
\hline $\mathrm{X} 1-\mathrm{X} 9$ & 46.5005 & 0.0045159 \\
\hline $\mathrm{X} 1-\mathrm{X} 10$ & 1.57386 & 0.0355173 \\
\hline $\mathrm{X} 1-\mathrm{X} 11$ & 27.4589 & 0.0231471 \\
\hline $\mathrm{X} 1-\mathrm{X} 12$ & 38.3113 & 0.0382758 \\
\hline $\mathrm{X} 1-\mathrm{X} 13$ & 39.3052 & 0.0080928 \\
\hline $\mathrm{X} 1-\mathrm{X} 14$ & 46.2861 & 0.0331327 \\
\hline
\end{tabular}

Table 3: Correlation Coefficient $\alpha^{2}=0.67$

\begin{tabular}{|c|c|c|}
\hline $\begin{array}{c}\text { Correlation } \\
\text { Between }\end{array}$ & $\begin{array}{c}\text { MSE Using } \\
\text { Least Squares }\end{array}$ & $\begin{array}{c}\text { MSE Using } \\
\text { Unbiased Ridge }\end{array}$ \\
\hline $\mathrm{X} 1, \mathrm{X} 2$ & 28.3239 & 0.0295791 \\
\hline $\mathrm{X} 1-\mathrm{X} 3$ & 26.6763 & 0.0084244 \\
\hline $\mathrm{X} 1-\mathrm{X} 4$ & 38.7922 & 0.0066016 \\
\hline $\mathrm{X} 1-\mathrm{X} 5$ & 61.6469 & 0.015094 \\
\hline $\mathrm{X} 1-\mathrm{X} 6$ & 42.9897 & 0.0242642 \\
\hline $\mathrm{X} 1-\mathrm{X} 7$ & 37.0921 & 0.0174133 \\
\hline $\mathrm{X} 1-\mathrm{X} 8$ & 59.5534 & 0.0087105 \\
\hline $\mathrm{X} 1-\mathrm{X} 9$ & 91.8063 & 0.0000733 \\
\hline $\mathrm{X} 1-\mathrm{X} 10$ & 38.3784 & 0.0059245 \\
\hline $\mathrm{X} 1-\mathrm{X} 11$ & 44.8721 & 0.0023228 \\
\hline $\mathrm{X} 1-\mathrm{X} 12$ & 66.4587 & 0.0110501 \\
\hline $\mathrm{X} 1-\mathrm{X} 13$ & 69.5876 & 0.0075445 \\
\hline $\mathrm{X} 1-\mathrm{X} 14$ & 113.142 & 0.0031203 \\
\hline
\end{tabular}

\begin{tabular}{|c|c|c|}
\hline $\begin{array}{c}\text { Correlation } \\
\text { Between }\end{array}$ & $\begin{array}{c}\text { MSE Using } \\
\text { Least Squares }\end{array}$ & $\begin{array}{c}\text { MSE Using } \\
\text { Unbiased Ridge }\end{array}$ \\
\hline $\mathrm{X} 1, \mathrm{X} 2$ & 35.6903 & 0.0079587 \\
\hline $\mathrm{X} 1-\mathrm{X} 3$ & 34.3758 & 0.0061240 \\
\hline $\mathrm{X} 1-\mathrm{X} 4$ & 57.3043 & 0.0004140 \\
\hline $\mathrm{X} 1-\mathrm{X} 5$ & 115.0238 & 0.042486 \\
\hline $\mathrm{X} 1-\mathrm{X} 6$ & 74.0384 & 0.0159949 \\
\hline $\mathrm{X} 1-\mathrm{X} 7$ & 64.4310 & 0.0030147 \\
\hline $\mathrm{X} 1-\mathrm{X} 8$ & 107.380 & 0.0007958 \\
\hline $\mathrm{X} 1-\mathrm{X} 9$ & 189.863 & 0.0025083 \\
\hline $\mathrm{X} 1-\mathrm{X} 10$ & 68.1341 & 0.0057765 \\
\hline $\mathrm{X} 1-\mathrm{X} 11$ & 83.3222 & 0.0018858 \\
\hline $\mathrm{X} 1-\mathrm{X} 12$ & 125.433 & 0.0037824 \\
\hline $\mathrm{X} 1-\mathrm{X} 13$ & 132.940 & 0.0039350 \\
\hline $\mathrm{X} 1-\mathrm{X} 14$ & 259.746 & 0.0037324 \\
\hline
\end{tabular}

Table 2: Correlation Coefficient $\alpha^{2}=0.51$

\begin{tabular}{|c|c|c|}
\hline $\begin{array}{c}\text { Correlation } \\
\text { Between }\end{array}$ & $\begin{array}{c}\text { MSE Using } \\
\text { Least Squares }\end{array}$ & $\begin{array}{c}\text { MSE Using } \\
\text { Unbiased Ridge }\end{array}$ \\
\hline $\mathrm{X} 1, \mathrm{X} 2$ & 26.2279 & 0.0444524 \\
\hline $\mathrm{X} 1-\mathrm{X} 3$ & 24.3146 & 0.0111884 \\
\hline $\mathrm{X} 1-\mathrm{X} 4$ & 33.2860 & 0.0029957 \\
\hline $\mathrm{X} 1-\mathrm{X} 5$ & 45.0645 & 0.006178 \\
\hline $\mathrm{X} 1-\mathrm{X} 6$ & 33.4187 & 0.0064171 \\
\hline $\mathrm{X} 1-\mathrm{X} 7$ & 29.1076 & 0.0451311 \\
\hline $\mathrm{X} 1-\mathrm{X} 8$ & 44.4291 & 0.0554930 \\
\hline $\mathrm{X} 1-\mathrm{X} 9$ & 61.7260 & 0.0508783 \\
\hline $\mathrm{X} 1-\mathrm{X} 10$ & 29.6791 & 0.0113255 \\
\hline $\mathrm{X} 1-\mathrm{X} 11$ & 33.2142 & 0.0075011 \\
\hline $\mathrm{X} 1-\mathrm{X} 12$ & 47.9239 & 0.0162912 \\
\hline $\mathrm{X} 1-\mathrm{X} 13$ & 49.6498 & 0.0027323 \\
\hline $\mathrm{X} 1-\mathrm{X} 14$ & 68.5781 & 0.0129765 \\
\hline
\end{tabular}




\section{UNBIASED RIDGE AND LEAST SQUARES REGRESSION METHODS COMPARISON}

Table 5: Correlation Coefficient $\alpha^{2}=0.99$

\begin{tabular}{|c|c|c|}
\hline $\begin{array}{c}\text { Correlation } \\
\text { Between }\end{array}$ & $\begin{array}{c}\text { MSE Using } \\
\text { Least Squares }\end{array}$ & $\begin{array}{c}\text { MSE Using } \\
\text { Unbiased Ridge }\end{array}$ \\
\hline $\mathrm{X} 1, \mathrm{X} 2$ & 253.630 & 0.0064327 \\
\hline $\mathrm{X} 1-\mathrm{X} 3$ & 250.785 & 0.0011331 \\
\hline $\mathrm{X} 1-\mathrm{X} 4$ & 606.225 & 0.0098181 \\
\hline $\mathrm{X} 1-\mathrm{X} 5$ & 1645.7712 & 0.026003 \\
\hline $\mathrm{X} 1-\mathrm{X} 6$ & 974.1748 & 0.0078642 \\
\hline $\mathrm{X} 1-\mathrm{X} 7$ & 900.5158 & 0.0048502 \\
\hline $\mathrm{X} 1-\mathrm{X} 8$ & 1461.07 & 0.0158666 \\
\hline $\mathrm{X} 1-\mathrm{X} 9$ & 3049.69 & 0.0170372 \\
\hline $\mathrm{X} 1-\mathrm{X} 10$ & 976.803 & 0.0026540 \\
\hline $\mathrm{X} 1-\mathrm{X} 11$ & 1218.541 & 0.0011670 \\
\hline $\mathrm{X} 1-\mathrm{X} 12$ & 1787.02 & 0.0043619 \\
\hline $\mathrm{X} 1-\mathrm{X} 13$ & 1908.30 & 0.0040964 \\
\hline $\mathrm{X} 1-\mathrm{X} 14$ & 4586.19 & 0.0038209 \\
\hline
\end{tabular}

References

Casella, G., \& Berger, R. (2002). Statistical Inference, $2^{\text {nd }} E d$. USA: Duxbury.

Crouse, R., Chun, J., \& Hanumara, R. C. (1995). Unbiased ridge estimation with prior information and ridge trace. Communications in Statistics, 24(9), 2341-2354.

Wichern, D. W., \& Churchill, G. A. (1978). A comparison of ridge estimators. Technometrics, 20(3), 301-310.

Gunst, R. F., Webster, J. T., \& Mason, R. L. (1977). Biased estimation in regression: An evaluation using mean squared error. Journal of the American Statistical Association, 72(356), 616-628.

Horel, A. E., \& Kennard, R. W. (1970a). Ridge regression: Biased estimation for nonorthogonal problems. Technometrics, 12, 5583.

Mason, R. L., Gunst, R. F., \& Webster, J. T. (1977). Regression analysis and problems of multicollinearty. Communications in Statistics, 4(3), 279-292.
McDonald \& Galarneau. (1975). A Monte Carlo evaluation of some ridge type estimators. Journal of the American Statistical Association, 70, 407-416.

Murthy, K. P. N. (2003). An introduction to Monte Carlo simulation in statistical physics. India: Indira Gandhi Center for Atomic Research.

Pliskin, L. J. (1987). A ridge-type estimator and good prior means. Communications in Statistics, 16(12), 34273429.

Swindel, B. F. (1976). Good ridge estimators based on prior information. Communications in Statistics, A5(11), 10651075. 